\title{
Novel Isoforms of Dlg Are Fundamental for Neuronal Development in Drosophila
}

\author{
Carolina Mendoza, ${ }^{1}$ Patricio Olguín, ${ }^{1}$ Gabriela Lafferte, ${ }^{1}$ Ulrich Thomas, ${ }^{2}$ Susanne Ebitsch, ${ }^{2}$ Eckart D. Gundelfinger, ${ }^{2}$ \\ Manuel Kukuljan ${ }^{1}$ and Jimena Sierralta ${ }^{1}$ \\ ${ }^{1}$ Programa de Fisiología y Biofísica, Instituto de Ciencias Biomédicas, Facultad de Medicina, Universidad de Chile, Santiago, Chile 6530499, and ${ }^{2}$ Leibniz \\ Institute for Neurobiology, D-39118 Magdeburg, Germany
}

\begin{abstract}
Drosophila discs-large ( $\mathrm{dlg}$ ) mutants exhibit multiple developmental abnormalities, including severe defects in neuronal differentiation and synaptic structure and function. These defects have been ascribed to the loss of a single gene product, Dlg-A, a scaffold protein thought to be expressed in many cell types. Here, we describe that additional isoforms arise as a consequence of different transcription start points and alternative splicing of $d l g$. At least five different $d l g$ gene products are predicted. We identified a subset of $d l g$-derived cDNAs that include novel exons encoding a peptide homologous to the $\mathrm{N}$ terminus of the mammalian protein SAP97/hDLG (S97N). Dlg isoforms containing the $\mathrm{S} 97 \mathrm{~N}$ domain are expressed at larval neuromuscular junctions and within the CNS of both embryos and larvae but are not detectable in epithelial tissues. Strong hypomorphic $d l g$ alleles exhibit decreased expression of S97N, which may account for neural-specific aspects of the pleiomorphic $d l g$ mutant phenotype. Selective inhibition of the expression of S97N-containing proteins in embryos by double-strand RNA leads to severe defects in neuronal differentiation and axon guidance, without overt perturbations in epithelia. These results indicate that the differential expression of $d l g$ products correlates with distinct functions in non-neural and neural cells. During embryonic development, proteins that include the S97N domain are essential for proper neuronal differentiation and organization, acting through mechanisms that may include the adequate localization of cell fate determinants.
\end{abstract}

Key words: dlg; SAP97; alternative transcripts; neuronal differentiation; scaffold proteins; dsRNA; Drosophila

\section{Introduction}

Intercellular communication requires the recruitment of signaling components to distinct subdomains at the plasma membrane. This allows specific arrangement of interacting proteins and contributes to the fidelity and efficiency of signaling events. Central to the assembly of these complexes is the expression of proteins that act as molecular scaffolds by providing multiple sites for protein-protein interactions, including among them PDZ [postsynaptic density 95 (PSD-95)/Discs large (Dlg)/zona ocludens-1 (ZO-1)] domains. Membrane-associated guanylate kinases (MAGUKs), such as the Drosophila Dlg-A protein and its mammalian relatives SAP90 (synapse-associated protein-90)/ PSD-95, SAP102/NE-dlg, Chapsyn-110/PSD-93, and SAP97/human DLG (hDLG), belong to this class of proteins. MAGUKs participate in the proper organization of neural signal transduction cascades (Garner et al., 2000) and are involved in the polarized organization of membrane components in epithelia (Tepass et al., 2001).

Dlg-A as encoded by the tumor suppressor gene $d l g$ contains

\footnotetext{
Received 0ct. 28, 2002; revised 0ct. 28, 2002; accepted Nov. 29, 2002.

This work was supported by Fondo Nacional de Desarrollo Científico y Tecnológico Grant 1000824, Fundación G. Puelma, Fundación Andes, Iniciative Científica Milenio Grant P01-007, and DID (J.S.), the Human Frontier Science Program and Fonds der Chemischen Industrie (E.D.G.), and Comisión Nacional de Investigacion Científica y Tecnológica graduate scholarships (C.M. and P.O.). We thank Dr. P. Wappner for advice on embryo injections and A. Figueroa and F. Vergara for excellent technical assistance.

Correspondence should be addressed to Jimena Sierralta, Programa de Fisiología y Biofísica, Instituto de Ciencias Biomédicas, Facultad de Medicina, Universidad de Chile, Independencia 1027, Santiago, Chile 6530499. E-mail: jsierra@@machi.med.uchile.cl.

Copyright $\odot 2003$ Society for Neuroscience $\quad$ 0270-6474/03/232093-09\$15.00/0
}

three PDZ domains, an Src homology 3 (SH3) domain, and a guanylate kinase-like domain (Woods and Bryant, 1991). Dlg-A localizes to epithelial septate junctions, in which it is required for the maintenance of apicobasal polarity; mutations in $\mathrm{dlg}$ cause, besides the lost of apicobasal polarity, tumorous overgrowth of larval imaginal disc epithelia (Woods et al., 1996). Moreover, $d l g$ gene products participate in the organization of neuromuscular junctions (NMJs) and in the establishment of asymmetric cell division (Lahey et al., 1994; Budnik et al., 1996; Ohshiro et al., 2000; Peng et al., 2000; Bellaiche et al., 2001). The complexity of Dlg functions is explained in part by its ability to interact with multiple proteins, such as ion channels, cell adhesion molecules, and other scaffolding molecules (Tejedor et al., 1997; Thomas et al., 1997a; Zito et al., 1997; Bellaiche et al., 2001; Mathew et al., 2002). SAP97/hDLG is also expressed in both neurons and epithelia and complements loss of $d l g$ functions during heterologous expression in flies (Müller et al., 1995; Thomas et al., 1997a,b). In Caenorhabditis elegans, the Dlg/SAP97 homolog, DLG-1 participates in the establishment of intercellular unions in epithelia (Firestein and Rongo, 2001).

Despite the diversity of $d l g$ functions, the primary structures of only two closely related gene products denoted as Dlg-A [960 and 975 amino acid (aa) residues, respectively], have been considered so far (Woods and Bryant, 1991; Hough et al., 1997). However, Northern analysis demonstrates the presence of at least five transcripts in the adult fly and in larvae (Woods and Bryant, 1989), and Western blot analysis points to the existence of several gene products (Lahey et al., 1994; Woods et al., 1996; Koh et al., 1999). We identified multiple 
alternatively processed transcripts of the $d l g$ gene, containing previously uncharacterized exons that map upstream of the region encoding Dlg-A and that encode a domain homologous to the $\mathrm{N}$ terminus of mammalian SAP97 (S97N). We show here that $d l g$ products containing this domain are predominantly expressed in neuropil regions of the CNS and at NMJs in late embryos and larvae. Selective inhibition of the expression of the forms that include the S97N domain by RNA interference results in the perturbation of normal development of embryonic neural tissues, with preservation of the expression of other $d l g$ products and epithelial integrity.

\section{Materials and Methods}

Flies. Flies were kept at $24^{\circ} \mathrm{C}$ on standard medium. The following $d l g$ mutant alleles were used: $d l g^{X I-2}, d l g^{m 30}, d l g^{v 59}$, and $d l^{m 52}$ (Woods et al., 1996). Strain $w^{1118}$ was used as a dlg wild-type control.

Antibodies. To generate antibodies specific for the S97N domain (anti$\mathrm{Dlg}_{\mathrm{S} 97 \mathrm{~N}}$ ) a sequence of $410 \mathrm{bp}$, starting with the first ATG of the open reading frame of expressed sequence tag (EST) clone LP07807, was subcloned into the pGEX5x-3 plasmid (Amersham Biosciences, Piscataway, $\mathrm{NJ}$ ). The GST (glutathione $S$-transferase) fusion protein was expressed in Escherichia coli, purified using glutathione beads (Sigma, St. Louis, MO), and injected in rabbits. The serum was affinity purified (Affigel-10; BioRad, Hercules, CA) using the fusion protein against which the antibody was raised. The rabbit polyclonal anti-Dlg $\operatorname{PDZ1-2}_{2}$ antibody (PDZ1-PDZ2 of Dlg-A) (Koh et al., 1999) was kindly provided by Dr. V. Budnik (University of Massachusetts at Amherst, Amherst, MA) and monoclonal antibody (mAb) 1D4 [anti-Fasciclin II (Fas II)] was a gift from Dr. C. Goodman (University of California Berkeley, Berkeley, CA). The mAbs BP102 (Seeger et al., 1993), Elav-7E8A10 (Ellis et al., 1993), and 22C10 (Zipursky et al., 1984) were obtained from the Developmental Studies Hybridoma Bank (University of Iowa, Iowa City, IA).

Western blot analysis. Body walls, brains, and imaginal discs were dissected out from late third-instar larvae, in ice-cold buffer (in mM: 128 $\mathrm{NaCl}, 2 \mathrm{KCl}, 4 \mathrm{MgCl}, 5 \mathrm{HEPES}, 35.5$ sucrose, and 5 EGTA). Both embryonic and larval tissues were homogenized in $100 \mu$ l of buffer ( $50 \mathrm{~mm}$ Tris, $25 \mathrm{~mm} \mathrm{KCl}, 2 \mathrm{~mm}$ EDTA, $0.3 \mathrm{~m}$ sucrose, and $2 \% \mathrm{SDS}$ ) at $80^{\circ} \mathrm{C}$. Proteins were separated in $10 \%$ gels by SDS-PAGE, transferred to nitrocellulose or nylon membranes, and incubated with anti-Dlg $\operatorname{PDZ1-2}_{\text {PDZ }}(1: 10,000)$ or anti$\operatorname{Dlg}_{S 97 \mathrm{~N}}(1: 1000)$ antibodies. Bands were visualized using a horseradish peroxidase (HRP)-conjugated goat anti-rabbit secondary antibody (Jackson ImmunoResearch, West Grove, PA) and a chemiluminescence assay (ECL Lumigen PS-3 Detection Reagent; Amersham Biosciences).

Immunohistochemistry. Staged embryos were dechorionated with bleach, fixed for $20 \mathrm{~min}$ in $4 \%$ formaldehyde in PBS-N-heptane (1:1), and devitellinized by treatment with methanol. Late third-instar larvae were dissected in calcium-free saline medium (in mM: $128 \mathrm{NaCl}, 2$ $\mathrm{KCl}, 4 \mathrm{MgCl}_{2}$, 5 HEPES, 35.5 sucrose, and $5 \mathrm{~mm}$ EGTA). The samples were fixed in nonalcoholic Bouin's solution (prepared by mixing 25 $\mathrm{ml}$ of formaldehyde from a $37.7 \%$ stock solution, $5 \mathrm{ml}$ of glacial acetic acid, and $75 \mathrm{ml}$ of saturated picric acid solution) for $1 \mathrm{hr}$ or in $4 \%$ paraformaldehyde for $30 \mathrm{~min}$. After fixation, specimens were washed in PBS containing $0.1 \%$ Triton $\mathrm{X}-100$. Primary antibody incubations were performed overnight at $4^{\circ} \mathrm{C}$; the anti-Dlg $\operatorname{PDZ1-2}_{\mathrm{PDZ}}$ antibody was used 1:2000 (embryos) or 1:10,000 (larvae), and anti-Dlg $\operatorname{siplN}_{\mathrm{N}}$ was diluted 1:200 for embryos and larvae. As secondary antibodies, a fluorescein-conjugated goat anti-horseradish peroxidase antibody was used at a 1:150 dilution, and a rhodamine-conjugated goat antirabbit antibody (Jackson ImmunoResearch) was used at 1:2000 dilution; confocal imaging was performed with a Zeiss (Oberkochen, Germany) LSM410 microscope or a Leica (Nussloch, Germany) DM LFSA microscope.

Double-strand RNA interference. The $1.2 \mathrm{~kb}$ fragment resulting from the EcoRV-BamHI digestion of EST clone LD33841 was subcloned into pBluescript SK ${ }^{-}$(Stratagene, La Jolla, CA). This fragment includes $802 \mathrm{bp}$ of $5^{\prime}$ untranslated region and $414 \mathrm{bp}$ encoding the S97N domain (exons A, 1, 3, 4, and 6). RNA was synthesized using T3 and T7 RNA polymerases after appropriate linearization of the plasmid. Preparation and injection of double-strand RNA (dsRNA) was performed according to Kennerdell and Carthew (1998). Embryos were collected over 30 min periods, dechorionated, and attached to a coverslip. After allowing partial desiccation, embryos were covered with halocarbon oil 700 (Sigma) and injected at the syncytial blastoderm stage with a volume equivalent to approximately one-third of the total volume of the embryo, using a Picopump injector (World Precision Instruments, Sarasota, FL). Embryos were allowed to develop until the appropriate stage and then fixed for immunohistochemical examination. The embryos were examined under a binocular scope using $45 \times$ magnification. Positive immunoreactivity is defined as any noticeable staining observed at this magnification. Major defects were considered as patterning abnormalities detectable under these conditions (binocular scope, $45 \times$ magnification).

\section{Results \\ Diversity of $d l g$ products originates from alternative transcriptional initiation and splicing}

To assess the diversity of Dlg isoforms, we performed a detailed sequence analysis of $d l g$-related EST clones. In addition to clones that correspond to Dlg-A (e.g., LD10659), we identified 12 EST clones that share a sequence encoding a protein domain with high homology ( $60 \%$ identity, $80 \%$ similarity) to the $\mathrm{N}$-terminal 66 aa residues of SAP97 (Fig. $1 A$ ) We refer to this region as $\mathrm{S} 97 \mathrm{~N}$ domain. The EST clones can be further divided into three groups, which are represented by clones RE30579, GH01107, and LD33841 (Fig. 1B). All three groups contain a poly(A) tail. RE30579 and LD33841 share a long 5' untranslated sequence encoded by exons $\mathrm{A}$ and most of the exon 1 of the $d \mathrm{lg}$ gene. RE30579 encodes a protein of 747 aa consisting of S97N, followed by PDZ1 to PDZ3 and the SH3 domain. It does not include the GUK domain and instead contains sequences encoded by exons 18-20 (Fig. $1 B$ ) and terminates with a potential PDZ-binding motif ( - SSI). The protein deduced from clone GH01107 (824 aa) exhibits an N-terminal region of 312 aa interspersed with several PEST sequences, which might promote protein degradation (Rechsteiner and Rogers, 1996). This unique $\mathrm{N}$ terminus is followed by S97N, PDZ1, and PDZ2 and then is spliced to exons 18-20 ending with the same C-terminal -SSI motif as clone RE30579 (Fig. 1B). Because of the absence of in-frame stop codons preceding the first ATG, we cannot rule out the existence of additional translational start sites that may be situated farther upstream. LD33841 represents a group of at least three ESTs obtained from different libraries of embryonic or larval origin (LD33841, LP07807, and RE57748). They encode a protein of 203 aa that includes $S 97 \mathrm{~N}$ and then is followed by a sequence completely unrelated to Dlg-A (Fig. 1B).

None of the EST clones corresponded to an isoform that could be aligned to the known vertebrate SAP97 proteins or to C. elegans DLG-1 in their entire length, i.e., from the S97N through the GUK domain. To examine whether a SAP97-like isoform exists in Drosophila, we performed a reverse transcription (RT)PCR analysis on embryonic total RNA. Using primers specific for the S97N domain and the C-terminal GUK domain, we obtained a product that encodes S97N, PDZ1, PDZ2, PDZ3, SH3, and GUK domains within a single protein, similar to SAP97. We refer to it as Dlg-S97 (Fig. $1 B$ ). Aside from its $\mathrm{N}$ terminus, this isoform also deviates from Dlg-A by the inclusion of additional 8 aa preceding PDZ1 (encoded by exon 9), by lacking a stretch of 160 aa between PDZ2 and PDZ3 (encoded by exon 12) (Fig. 1C) and by the presence of short aa stretches within the C-terminal half of the so-called HOOK region between the SH3 and GUK domains (encoded by exons 18 and 19) (for a detailed illustration, see Fig. 
A

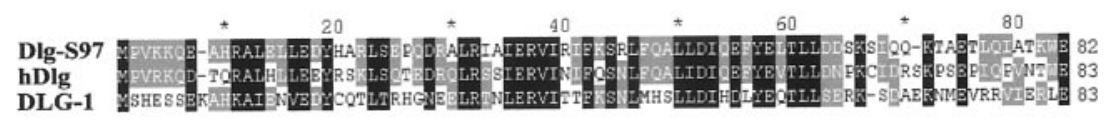

B

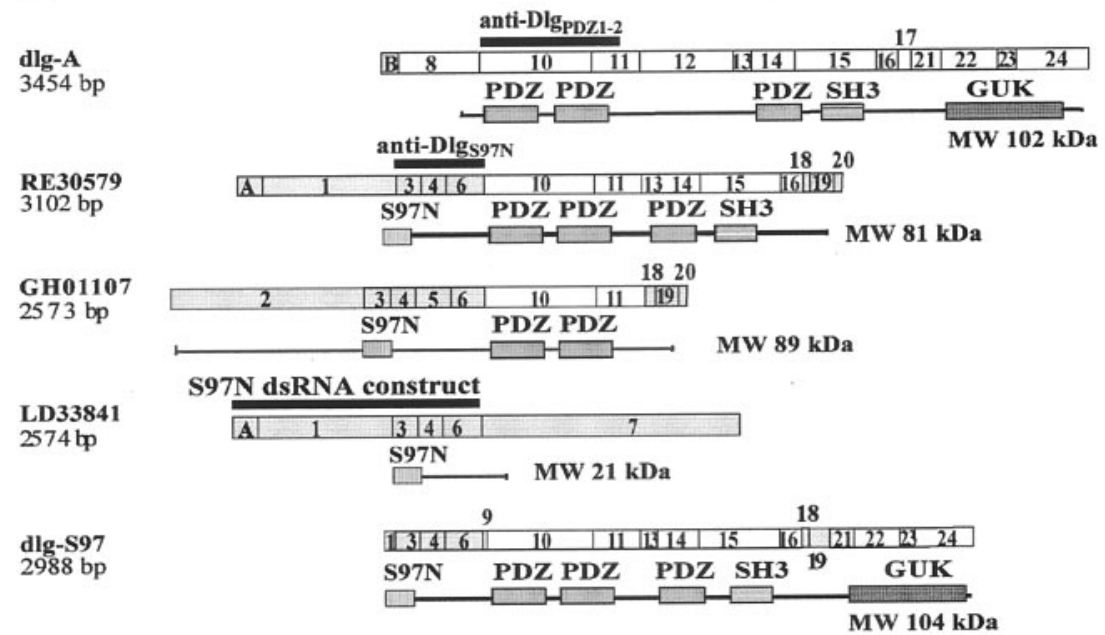

C

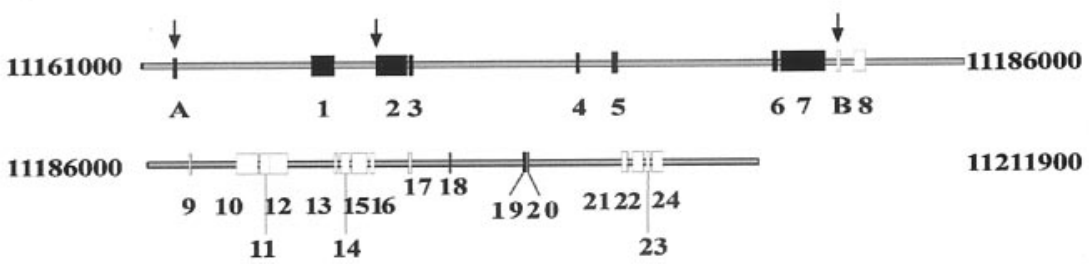

Figure 1. Novel isoforms originate from the $\mathrm{dlg}$ locus. A, Alignment of the predicted S97N domain of Drosophila melanogaster (Dlg-S97), Homo sapiens (hDlg), and C. elegans (DLG-1). Identical amino acids are highlighted in black, and similar residues are highlighted in gray. B, Exon composition of dlg transcripts as deduced from EST and RT-PCR cDNA analyses, including Dlg-A. Each numbered box represents a predicted translated exon, and lettered boxes depict predicted untranslated exons. Exon numbering follows the 5' to $3^{\prime}$ disposition of the coding strand in the genomic sequence (see C); shadowed boxes indicate the novel sequences that are absent in the Dlg-A transcript. Predicted open reading frames (black line), protein domain structures (patterned boxes), and calculated molecular weights (MW) of the proteins are shown below each transcript plot. Overlying lines mark the regions recognized by the antibodies used throughout this work, and the region used for the dsRNA interference experiments described below (see Materials and Methods). C, Genomic structure of $d l g$. Black boxes indicate exons absent in the Dlg-A transcript. Vertical arrows mark the putative positions of transcriptional start sites as deduced from the $5^{\prime}$ ends of the various groups of EST and CDNA clones. Note that exons 11-12 and 19-20 have no intervening intronic sequence between them.

$1 B, C)$. From this analysis, it can be concluded that at least five different isoforms, including Dlg-A, are predicted to originate from the $d l g$ locus (Fig. $1 B$ ).

Our analysis indicates that the genomic organization of the $\mathrm{dlg}$ locus is more complex than reported previously (Woods and Bryant, 1991). Alignment of the sequences of dlg-related ESTs and our RT-PCR products with the corresponding genomic region (provided by the Fly Genome Project; Myers et al., 2000) reveals that $d l g$ spans a region of $\sim 37 \mathrm{~kb}$, thereby comprising at least 26 exons (Fig. 1C). On the basis of this new data, we propose a revised nomenclature for $d l g$ exons (Fig. $1 B, C$ ). Eight exons (Fig. $1 C$, exons $A$ and $1-7$ ) map upstream of the $5^{\prime}$ nontranslated exon of Dlg-A (Fig. $1 C$, exon $B$ ) and primarily correspond to a conceptual gene annotated as CG1730 in the Fly Genome Project. Between the exons encoding the SH3 and GUK domains, three additional exons (exons 18-20) are found, which encode the alternative C terminus associated with ESTs RE30579 and GH01107. Notably, transcripts encoding S97N-containing iso- forms are initiated at least $20 \mathrm{~kb}$ upstream of those coding for the previously described Dlg-A protein.

\section{Differential expression of $d l g$ products during Drosophila development}

To explore the expression of proteins that contain the S97N domain, we generated antibodies against a fusion protein containing this domain (anti-Dlg ${ }_{S 97 N}$ ) (Fig. $1 B)$. We used this antibody and a previously described polyclonal antibody against the PDZ1 and PDZ2 domains (anti-Dlg $\operatorname{lozZ1-2}_{\text {) }}$ (Koh et al., 1999) for comparative immunocytochemical and Western blot analyses. In addition, we considered information reported by Woods and Bryant (1991) using an antiDlg ${ }_{\mathrm{SH} 3-\mathrm{GUK}}$ antibody.

Western blot analysis using anti$\mathrm{Dlg}_{\mathrm{S} 97 \mathrm{~N}}$ antibodies revealed single prominent bands of $\sim 116 \mathrm{kDa}$ in body wall muscle extracts and of $\sim 130 \mathrm{kDa}$ in homogenates from embryos and larval brains (Fig. $2 A$ ). Similar molecular weight bands were also detected by anti-DlgPDZ1-2 (Fig. $2 B$ ) antibodies. These bands may represent isoforms containing both S97N and PDZ domains or proteins with only one of these domains and similar molecular weights. Moreover, bands of similar if not identical molecular weights have been detected by anti-Dlg SHH-GUK $_{\text {S }}$ (Woods et al., 1996). To verify the existence of Dlg-S97 at the protein level, we took advantage of the fact that the $d l g^{v 59}$ mutant allele gives rise to truncated gene products attributable to a premature stop codon in the region encoding the GUK domain (Woods and Bryant, 1991). An immunoblot analysis of body wall muscle extracts of $d l^{\nu 59}$ mutant larvae revealed that the molecular weight of the anti$\mathrm{Dlg}_{\mathrm{S} 97 \mathrm{~N}}$-immunoreactive band decreased to $\sim 100 \mathrm{kDa}$ compared with the $116 \mathrm{kDa}$ protein expressed in wild type (Fig. 2C). This result is consistent with the presence of both the S97N and GUK domains in this prominent isoform; we therefore conclude that the $116 \mathrm{kDa}$ band corresponds to Dlg-S97. Whereas the 116 and $130 \mathrm{kDa}$ isoforms are recognized by both anti-Dlg ${ }_{597 \mathrm{~N}}$ and anti-Dlg $\operatorname{PDDZ1-2}_{2}$ antisera, other isoforms are immunoreactive to anti-Dlg $\mathrm{PDZl}-2$ only. This applies to a prominent band of $\sim 97 \mathrm{kDa}$ present in body wall muscle extracts and to a $\sim 120 \mathrm{kDa}$ band detected in wing imaginal discs. Remarkably, in the latter tissue, anti-Dlg ${ }_{597 \mathrm{~N}}$-positive bands are completely absent (Fig. 2, compare $A, B$ ). Whereas the exact domain composition of the $97 \mathrm{kDa}$ isoform remains elusive, the $120 \mathrm{kDa}$ imaginal disc isoform most likely corresponds to Dlg-A, because transgenically expressed Dlg-A yielded a band of the same size (Hough et al., 1997; Thomas et al., 2000). Therefore, it can be proposed that at least four different proteins may be products of the $\mathrm{dlg}$ locus. The occurrence of proteins of lower molecular weight such as those predicted from the EST clones cannot be ruled out by this analysis. Our Western blot experi- 


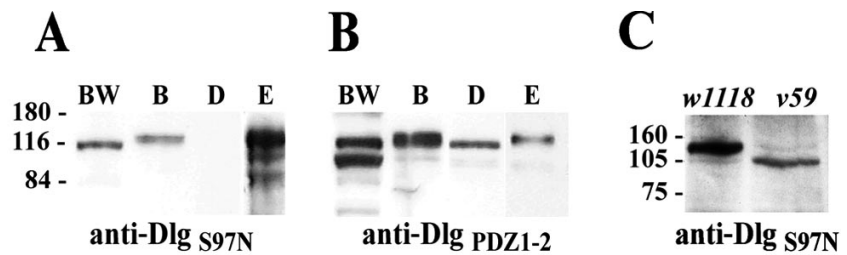

Figure 2. Expression of different Dlg isoforms in Drosophila. $A, B$, Western blot analysis of larval body wall muscles $(B W)$, larval brains $(B)$, wing imaginal discs $(D)$, and $0-20 \mathrm{hr}$ embryos $(E)$ probed with anti-Dlg $\mathrm{g}_{597 \mathrm{~N}}(A)$ and anti-Dlg $\operatorname{lopz1-2}(B)$. Molecular weight markers are the same for both panels. Note the absence of bands in wing imaginal discs in $A$. C, Western blot analysis of body wall extracts of wild-type and $\mathrm{dlg}^{\mathrm{v} 59}$ mutants probed with anti-Dlg $\mathrm{g}_{57 \mathrm{~N} \text { N }}$. Note the shift of the $116 \mathrm{kDa}$ band in the $\mathrm{dlg}^{\mathrm{v} 5}$ mutant.

ments reveal bands of lower molecular weight that could either represent degradation products of the larger isoforms or indicate the presence of these isoforms. Discrepancies between calculated and apparent molecular weights as well as small differences in molecular weights among proteins expressed at different developmental stages or in different tissues may reflect further complexity at the levels of posttranscriptional and posttranslational processing.

To further characterize the expression patterns of the S97Ncontaining proteins during Drosophila development, we performed immunohistochemical experiments focusing on embryonic stages and third-instar larvae. These experiments revealed that $\mathrm{S} 97 \mathrm{~N}$-containing proteins are expressed throughout embryogenesis. During the cellular blastoderm stage, forms containing S97N are localized to the cell borders, in a pattern similar to that detected by anti-Dlg $\operatorname{PDZ1-2}_{\mathrm{P}}$ antiserum (Fig. $3 A, B$ ), although the pattern observed with anti-D $\lg _{\mathrm{S} 97 \mathrm{~N}}$ consistently appeared to be more diffuse. From embryonic stage 11 onward, anti-Dlg $\lg _{597 \mathrm{~N}}$ immunoreactivity is found predominantly in the developing nervous system. Thus, strong labeling is observed in emerging axon bundles in the ventral cord and the brain (Fig. 3C,D, respectively). In the ventral cord neuropil, anti- $\mathrm{Dlg}_{\mathrm{S} 97 \mathrm{~N}}$ colocalizes with the axonal marker BP102 (Seeger et al., 1993) (Fig. 3E-G). In sharp contrast with the pattern observed with anti-Dlg $\operatorname{PDZ1-2}_{\text {, }}$ anti$\mathrm{Dlg}_{\mathrm{S} 97 \mathrm{~N}}$ antibodies do not label epithelial tissues at these late embryonic stages (Fig. $3 \mathrm{H}, \mathrm{I}$ ). In embryos of stages 14 and 15, anti-Dlg $\operatorname{Sg}_{\mathrm{S} 7 \mathrm{~N}}$ immunoreactivity is also present in the developing muscle, as can be seen in Figure 3, $E$ and $I$.

Dlg is known to be an important scaffolding protein for cell membrane proteins, including Shaker potassium channels and Fas II cell adhesion molecules at type I boutons of the NMJ. This was best studied in body wall muscles of third-instar larvae (Tejedor et al., 1997; Thomas et al., 1997a; Zito et al., 1997). To examine which isoforms may be involved in these functions, we first explored the expression of the $\mathrm{S} 97 \mathrm{~N}$ domain in body wall muscles and found that it is highly concentrated at type I boutons of NMJs. In fact, anti-Dlg S97N $_{\mathrm{N}}$ immunoreactivity at synaptic boutons is not distinguishable from anti-Dlg $\operatorname{PDZ1-2}$ staining (Fig. $4 A, B)$. This colocalization indicates that $597 \mathrm{~N}$ isoforms are enriched postsynaptically within the subsynaptic reticulum (cf. Lahey et al., 1994). A possible association of S97N isoforms with presynaptic bouton membranes cannot be resolved at the light microscopic level. The presence of anti-Dlg $\mathrm{Dg}_{97 \mathrm{~N}}$ immunoreactivity in motoneuronal axons (Figs. 3C, $4 D$ ), however, suggests that at least one $\mathrm{S} 97 \mathrm{~N}$-containing isoform is also localized presynaptically. In the larval CNS, anti-Dlg $\operatorname{s97N}_{\mathrm{N}}$ labeling remains enriched within neuropil regions of ventral ganglia and the brain, as well as in the emerging axon bundles (Fig. $4 C, D$, and data not shown). In
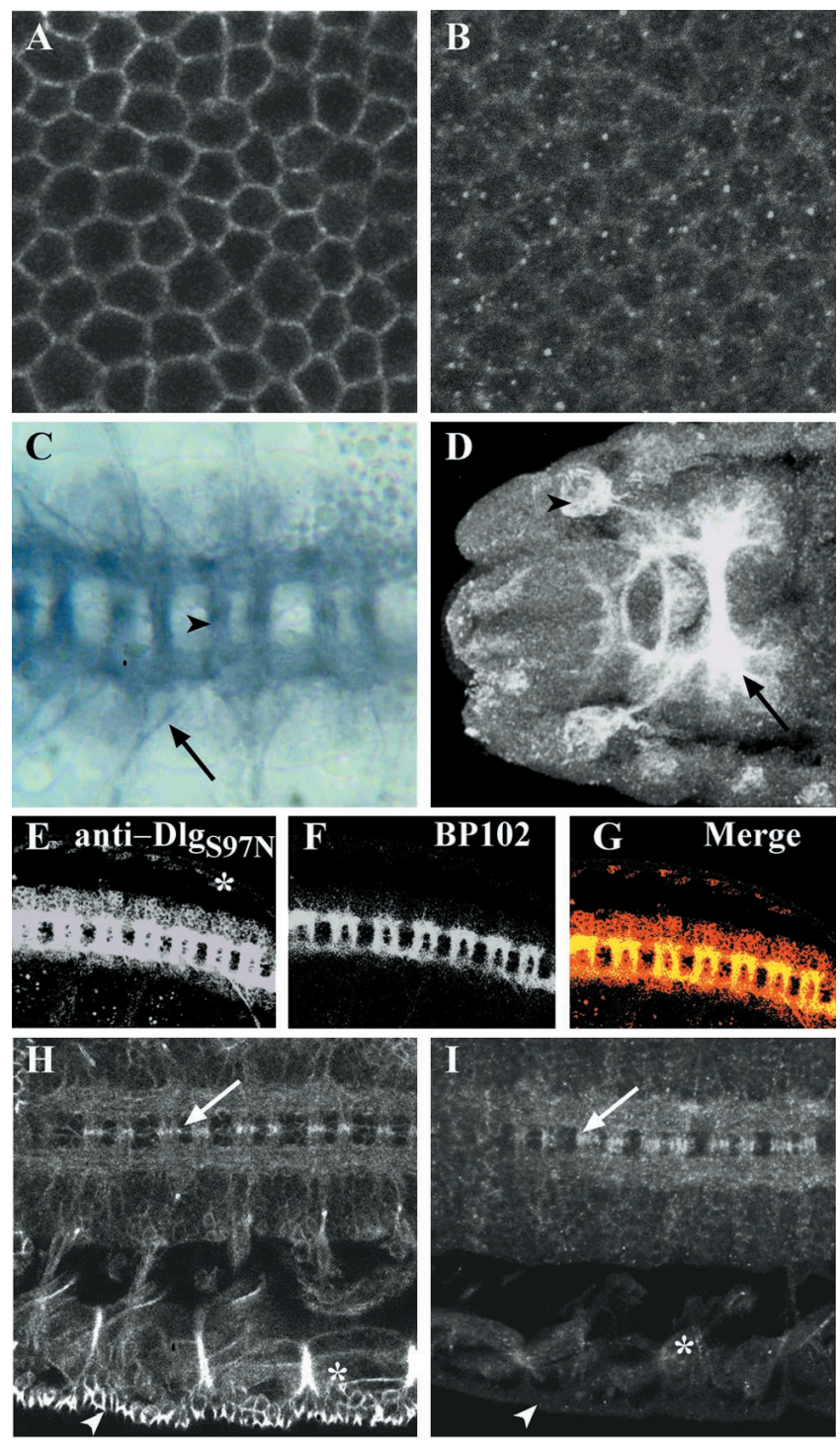

Figure 3. $597 \mathrm{~N}$-containing Dlg isoforms and Dlg-A are differentially expressed during embryogenesis. $A, B$, Superficial optical sections of stage 6 embryos stained with anti-Dlg $\operatorname{PDZ1-2}_{\mathrm{P}}(A)$ and anti-Dlg $\operatorname{sg}_{59 \mathrm{~N}}(B) . C$, Dorsal view of the ventral cord dissected out of a stage 14 embryo

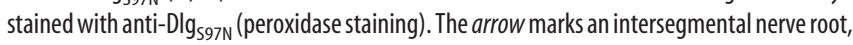
and the arrowhead indicates a commissural axon tract. D, Projection of serial confocal sections of the head of a stage 13 embryo (dorsal view) stained with anti-Dlg ${ }_{597 \mathrm{~N}}$. The arrow shows a brain lobe, and the arrowhead indicates an optic lobe placode. E, F, Confocal section of a stage 14 embryo stained with anti-Dlg $g_{597 \mathrm{~N}}$ and BP102 (which labels (NS axons). G, Merged view of $E$ and $F$, showing the colocalization in the neuropil. $H, I$, Confocal images of stage 15 embryos stained with anti-Dlg ${ }_{\mathrm{PDZ1}-2}$ and anti-Dlg $\mathrm{g}_{597 \mathrm{~N}}$. Arrows indicate ventral cords, arrowheads point to epidermal epithelia, and asterisks mark body wall muscles. Note the absence of anti-Dlg $g_{597 \mathrm{~N}} \mathrm{im}-$ munoreactivity in the epidermis and the presence of anti-Dlg $g_{597 \mathrm{~N}}$ immunoreactivity in muscles (also seen in $E$ ). Anterior is left in all panels.

line with our Western blot analysis, we did not detect S97N immunoreactivity in the epithelia of wing imaginal discs or salivary glands, both of which are epithelial tissues that are strongly labeled with anti-Dlg $\operatorname{PDZ1-2}_{\text {(Fig. }} 4 E-H$ ). We therefore conclude that $\mathrm{S} 97 \mathrm{~N}$ isoforms of Dlg are predominantly expressed in the nervous system and in body wall muscles.

Effects of mutations in $\mathrm{dlg}$ on synaptic expression of Dlg-S97 Mutations in $\mathrm{dlg}$ affect both structural and physiological properties of larval NMJs (Lahey et al., 1994; Budnik et al., 1996). In line with these findings, expression of Dlg at synaptic boutons, as 


\section{anti-Dlg $\operatorname{PDZ1-2}_{\text {P }}$}
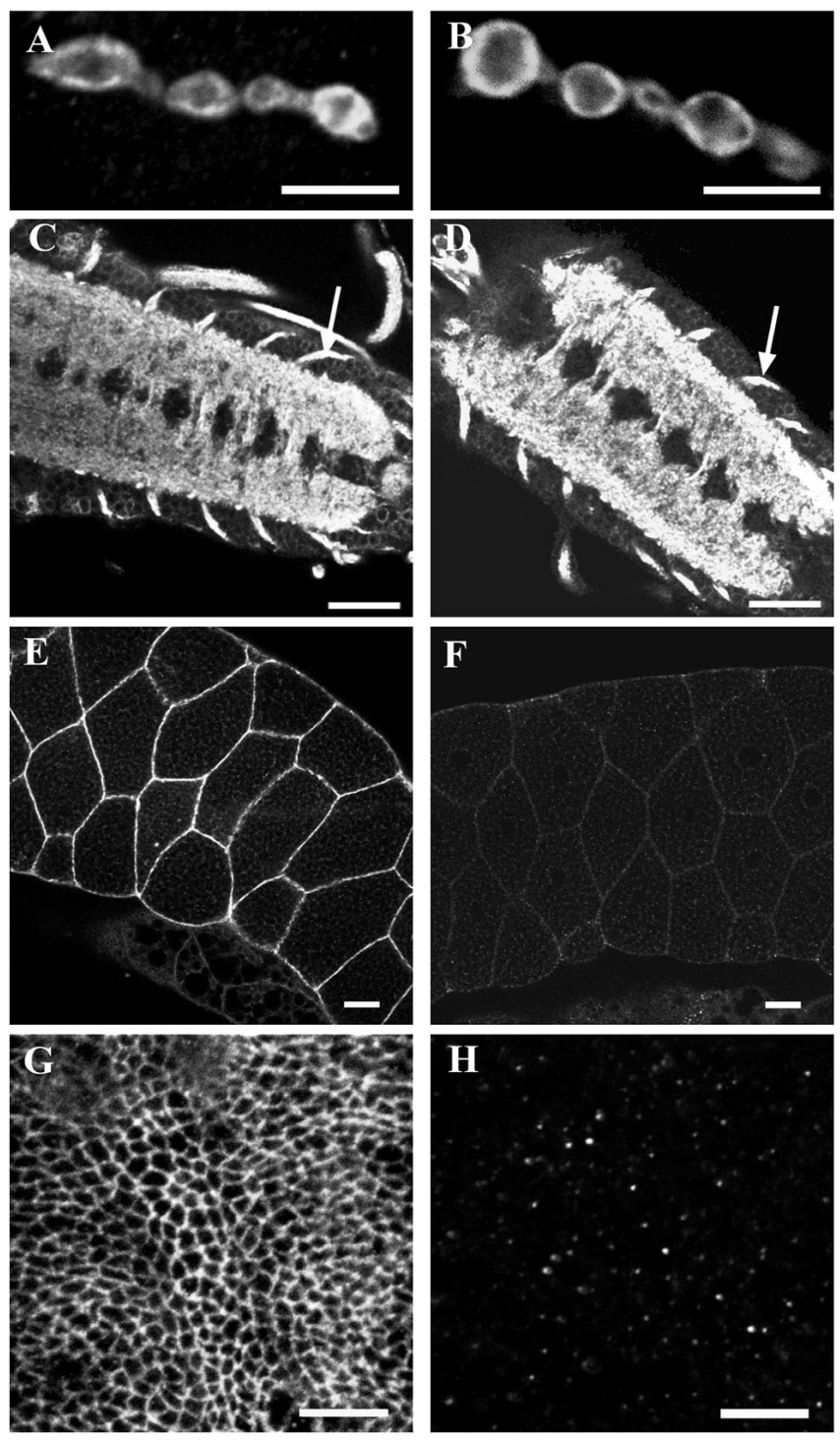

Figure 4. Differential expression of Dlg isoforms in larval tissues. Confocal images of tissues dissected out of third-instar larvae stained with anti-Dlg $g_{\text {PDz1-2 }}$ (left column) or anti-Dlg $g_{597 \mathrm{~N}}$ (right column). $A, B$, Type I synaptic boutons at body wall muscles. C, D, Ventral ganglia. Note the strong staining of axon bundles emerging from the ganglia (indicated by arrows). E, $F$, Salivary gland epithelia. $G, H$, Wing imaginal discs. Note the lack of $\operatorname{Dlg}_{597 \mathrm{~N}}$ staining in epithelial tissues $(F, H)$. Scale bars: $A, B, G, H, 10 \mu \mathrm{m} ; C-F, 25 \mu \mathrm{m}$.

revealed by anti-Dlg $\operatorname{PDZ1-2}_{\text {PD }}$ or anti-Dlg $\operatorname{SH}_{\mathrm{SH}-\mathrm{GUK}}$ immunoreactivities, is reduced in various mutant alleles (Lahey et al., 1994; Tejedor et al., 1997; Thomas et al., 1997a; Koh et al., 1999). To specifically address effects on the expression of $\mathrm{S} 97 \mathrm{~N}$ isoforms of

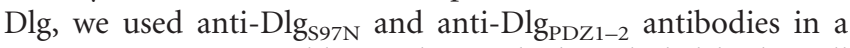
comparative Western blot analysis, which included body wall muscle extracts from $d l^{X I-2}, d l g^{m 52}$, and $d g^{m 30}$ mutant larvae (Fig. 5A,B). For both antibodies, immunoreactive bands detected in extracts from wild-type controls and $\mathrm{dlg}^{m 30}$ mutant larvae were indistinguishable, consistent with a single missense mutation associated with $\mathrm{dlg}^{m 30}$ (Woods et al., 1996). In contrast, anti-Dlg $\mathrm{S}_{\mathrm{S} 9 \mathrm{~N}}$-immunoreactive bands were found reduced to undetectable levels in extracts of both $d l^{X I-2}$ and $d g^{m 52}$ mutant larvae (Fig. 5B). On the other hand, low levels of size-reduced $d l g$ products could still be detected in $d l_{g}^{X I-2}$ mutants using anti-
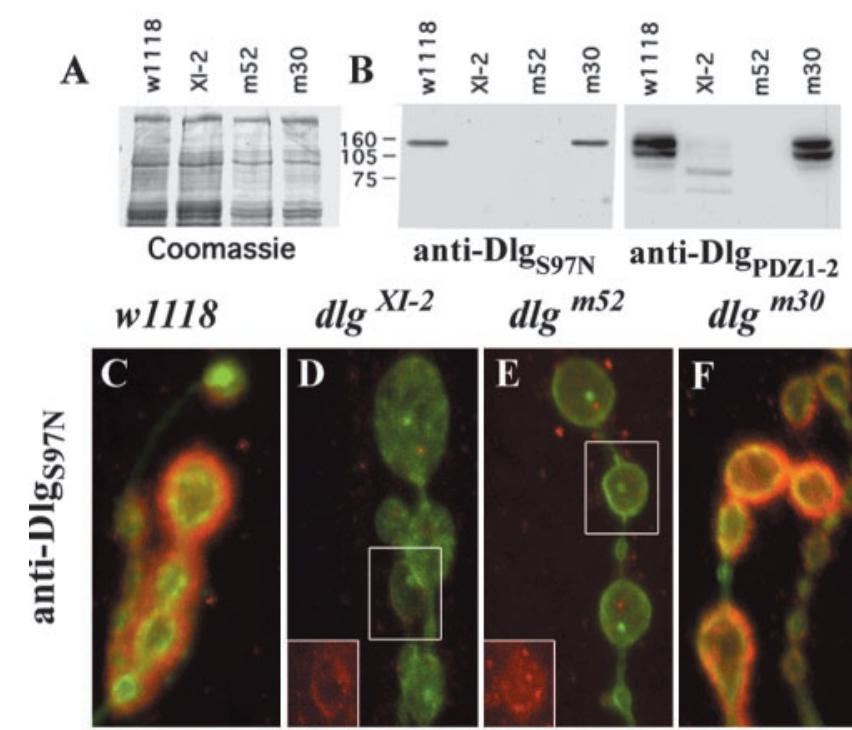

$\operatorname{dlg}^{m 52}$

dlg $^{m 30}$
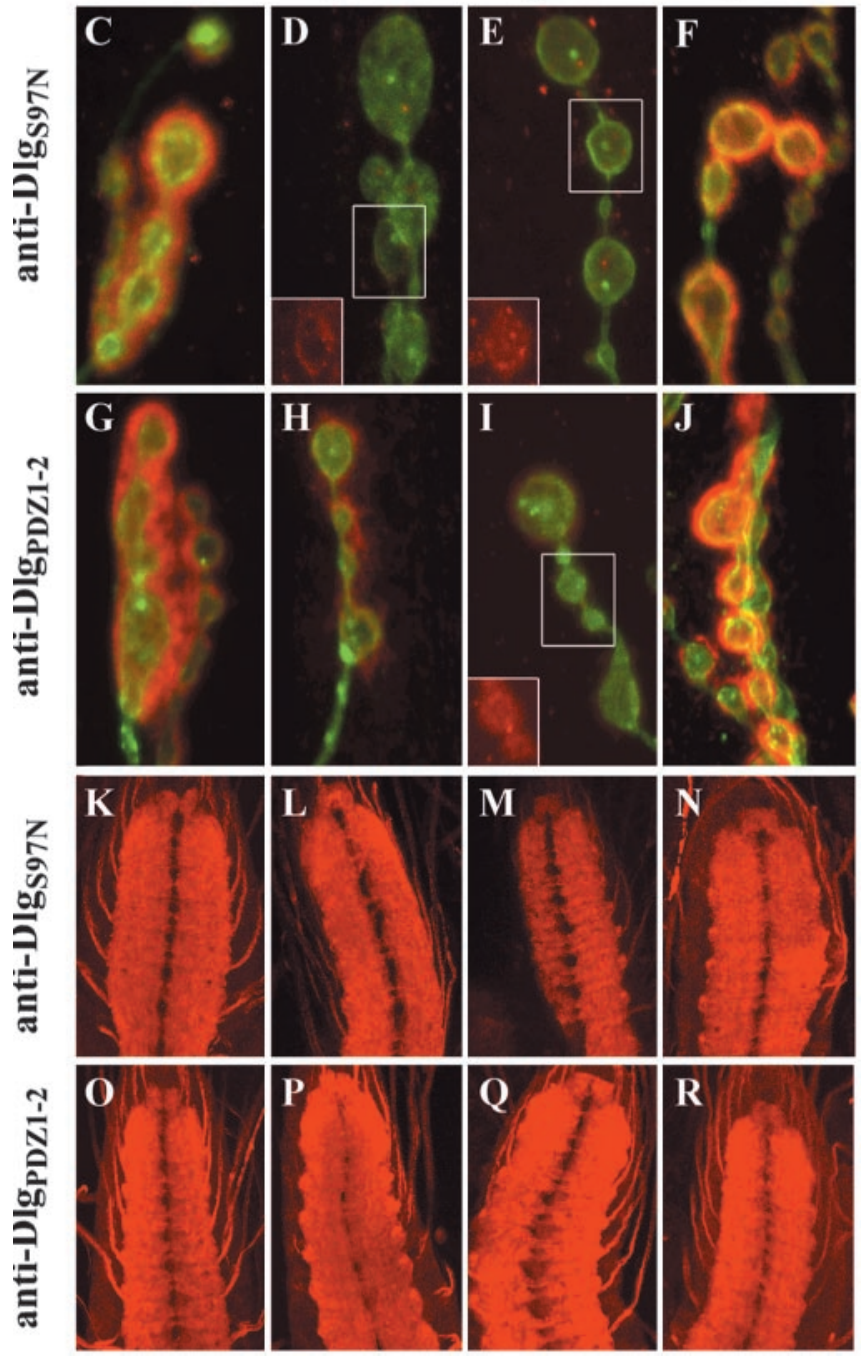

Figure 5. Expression of Dlg isoforms in dlg mutant larvae. $A, B$, Comparable amounts of body wall extracts from $\mathrm{dlg}^{+}$(w1118) and dlg mutant alleles XI-2, m52, and $m 30$ as revealed by Coomassie blue staining $(A)$ were subjected to Western blot analyses using anti-Dlg $g_{597 \mathrm{~N}}$ and anti-Dlg $\operatorname{lpDZ1-2}_{\text {PD }}(B) .(-J$, Type I boutons at NMJs on muscles 6 and 7 of $w 1118$ and $d l g$ mutant larvae. Nerve terminals as visualized by anti-HRP immunoreactivity (green) are primarily surrounded by anti-Dlg $\operatorname{PDZ1-2}_{2}(C-F)$ or anti-Dlg ${ }_{597 \mathrm{~N}}(G-J)$ immunoreactivities (red $)$. Selected boutons in $D, E$, and $/$ are shown as insets to document residual Dlg in the absence of anti-HRP labeling. All images represent merged Z-series of 25 confocal slices taken at $0.3 \mu \mathrm{m}$ steps and with identical settings. $K-R$, Dlg-specific immunoreactivities in ventral ganglia. All images are composed of 20 confocal slices taken at $1 \mu \mathrm{m}$ steps and with identical settings. Note that, for all mutant alleles, both antibodies yield considerable staining within the neuropil regions.

Dlg $_{\text {PDZ1-2 }}$ antibodies (Fig. 5B). In fact, immunofluorescent signals at synaptic boutons of both alleles, although strongly diminished compared with wild type (Fig. $5 C, G$ ) or $d l^{m 30}$ (Fig. 5F, J), were still obtained with either antibody (Fig. $5 D, H$ and $E, I$ ). We thus conclude that the mutations underlying $d l_{g}^{X I-2}$ or $d l_{g}^{m 52}$ (Woods et al., 1996) do not cause a complete absence of Dlgrelated proteins at NMJs. 
The perdurance of mutant $d l g$ gene products was even more evident in the larval CNS of all three mutant alleles, with both anti-Dlg $_{\mathrm{S} 97 \mathrm{~N}}$ and anti-Dlg $\operatorname{PDZ1-2}_{\mathrm{P}}$ antibodies yielding strong immunfluorescent signals (Fig. $5 K-R$ ). As in wild-type controls, Dlg-specific immunoreactivities remained highly enriched within neuropil regions of the mutant CNS, suggesting that the subcellular targeting of mutant Dlg isoforms is not severely affected. From these observations, we can conclude that neither $d l_{g}^{X I-2}$ nor $d l_{g}^{m 52}$ might constitute null alleles with regard to synaptic functions.

\section{S97N-containing proteins are required for normal neuronal differentiation}

To explore the functional role of the S97N-containing isoforms during embryonic development, we used the dsRNA interference technology to interfere with their expression in Drosophila embryos (Kennerdell and Carthew, 1998). The dsRNA was synthesized on the basis of sequences that occur only in the S97N domain-containing $d l g$ transcripts, i.e., those including exons $\mathrm{A}$, $1,3,4$, and 6 (Fig. $1 B$ ). Injection of S97N-dsRNA was effective in decreasing the expression of the proteins containing S97N, as demonstrated by immunofluorescence analysis of injected embryos. At stages $14-17$, only $5 \%$ of the embryos injected with the S97N-dsRNA displayed some anti-Dlg $\operatorname{Sg7N}_{\mathrm{N}}$ immunoreactivity $(n=203)$ compared with $73 \%$ of the buffer-injected embryos $(n=159)$ (Fig. $6 A, B)$. Immunostaining with anti-Dlg $\operatorname{PDZ1-2}_{\mathrm{PD}}$ in epithelia displayed a normal pattern, which is consistent with a preserved expression of the Dlg forms encoded by transcripts lacking the $S 97 \mathrm{~N}$ domain [81\% of embryos with positive immunohistochemistry $(n=84)$ compared with $93 \%$ of the bufferinjected embryos $(n=98)$ ] (Fig. $6 C, D)$. We failed to detect antiD $\lg _{\mathrm{PDZ} 1-2}$ immunoreactivity in the ventral cord of embryos injected with S97N-dsRNA.

We evaluated neuronal development in embryos injected with S97N-dsRNA by staining with anti-Elav-mAb 7E8A10, a marker for differentiated CNS and PNS neurons (Robinow and White, 1991), the anti-Futsch mAb 22C10, which selectively labels processes of all sensory neurons (Hummel et al., 2000), and anti-Fas II antibodies, which label a subpopulation of CNS axons (Grenningloh et al., 1991). The dsRNA-S97N injection is associated with major defects in neuronal development, as exemplified in Figure 6, $F, H$, and J. Alterations (major defects; see Materials and Methods) in the pattern of Elav immunoreactivity were observed in $32 \%$ of the embryos $(n=162)$ compared with $7 \%$ of major defects in buffer-injected embryos $(n=57)$ (Fig. $6 E, F)$. These defects included the loss of labeling of peripheral neurons, local or generalized expansion of Elav immunoreactivity into the ventral cord and the brain, or gross distortion of ventral cord architecture. Eighty-two percent of the embryos $(n=169)$ injected with S97N-dsRNA also exhibit marked defects in CNS and PNS axonal structure, as revealed by immunostaining with $\mathrm{mAb}$ $22 \mathrm{C} 10$ (Fig. $6 \mathrm{H}$ ). In contrast, only $4 \%$ of the buffer-injected embryos showed defects $(n=179)$. The observed defects range from the loss of the longitudinal and commissural patterns in the ventral cord to the severe misrouting of axons toward the periphery and the complete disorganization of the regular structure of central and peripheral processes (Fig. 6G,H). Immunostaining with anti-Fas II antibodies revealed additional defects of the axonal organization, including lack of commissural tracts and longitudinal fascicles and marked defects in axon branching, extension, and guidance. These defects were observed in 35\% of the examined embryos injected with S97N-dsRNA $(n=170)$, whereas

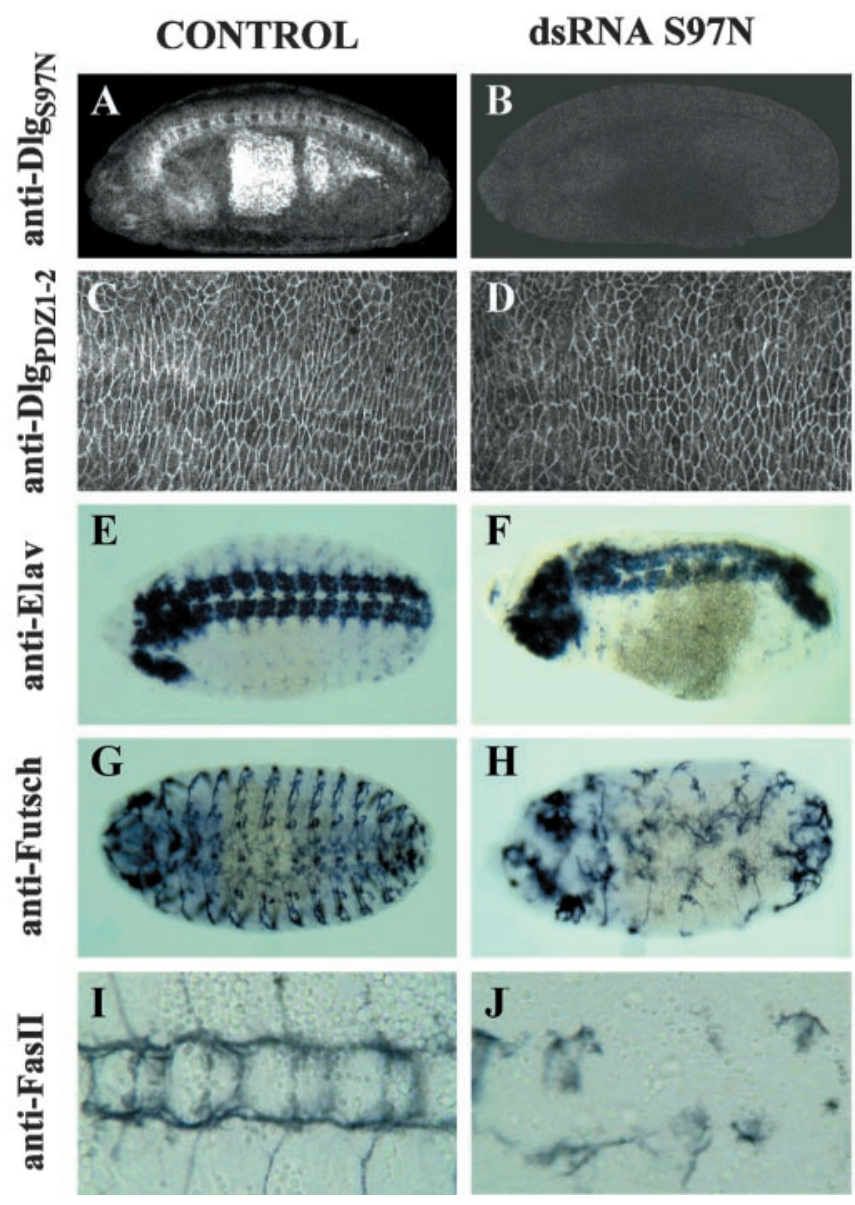

Figure 6. Targeted suppression of proteins containing the S97N domain severely affects neural development. Pairwise immunostainings of equally staged embryos injected with buffer (CONTROL) or S97N-double-strand RNA (dsRNA S97N). The antibodies used are indicated on the left. $A, B$, Confocal medial sections of stage 13 embryos showing that dsRNA-S97N effectively decreases anti-Dlg $g_{S 97 \mathrm{~N}}$ immunoreactivity. C, D, Confocal views of the superficial epithelium of stage 13 embryos demonstrating that injection of dsRNA-S97N does not affect anti-Dlg PDZ1-2 $_{2}$ immunoreactivity in this tissue. $E, F$, Ventral view of stage 13 embryos showing the disorganization of the Elav immunoreactivity in dsRNA-S97N-injected embryos. G, H, The highly ordered organization of CNS and PNS neurites as revealed by $22 \mathrm{C} 10$ immunoreactivity in wild type $(G)$ is disrupted during dsRNA-S97N injection $(H)$. $I$, J, The severe disorganization of axonal branching and routing associated with dsRNA-S97N injection is also evident for a subset of CNS neurons expressing Fasciclin II. E-H show peroxidase stainings of whole-mount embryos, and I and J show the dorsal aspect of ventral cords from dissected embryos. Anterior is left in all panels.

only $4 \%$ of the buffer-injected embryos displayed alterations of the Fas II immunoreactivity $(n=134)$ (Fig. 6I,J).

\section{Discussion}

\section{Multiple transcripts originate from the $d l g$ locus}

Initial genetic studies of the $d l g$ locus predicted a considerable degree of complexity of encoded products (Perrimon, 1988), and Northern analysis confirmed the existence of multiple $d l g$ transcripts (Woods and Bryant, 1989). Our data provide a molecular correlate for this anticipated complexity of the $d l g$ locus. Thus, the $d l g$ gene has alternative transcription initiation and splice sites, which lead to the expression of diverse transcripts and corresponding polypeptides. Not all identified ESTs could be assigned to protein products yet. However, except for GH01107, all EST cDNAs contain long open reading frames between in-frame stop codons and carry poly(A) stretches at their $3^{\prime}$ ends, suggesting that they may represent naturally occurring mRNAs. Similar forms of transcripts-ESTs were identified in embryonic, larval, 
and adult cDNA libraries (Flybase; J. Sierralta, unpublished data). In transcripts for isoforms that terminate with the -SSI motif encoded on exon 19, the protein coding region is followed by an untranslated region and a poly(A) stretch present in genomic DNA. Thus, their identity as physiologically relevant protein coding mRNAs remains unclear. On the other hand, we established that alternative transcripts of the dlg locus contain an S97N domain and demonstrated that variants containing this domain derive from different transcriptional start sites. Furthermore, we mapped the chromosomal location of several new exons encoding S97N-containing transcripts. The corresponding genomic region has been identified previously as an independent gene "CG1730" by the Drosophila Genome Project.

It should be noted that our results do not establish a correspondence between the variety of possible transcripts emerging from this work and a previous Northern analysis (Woods and Bryant, 1989). On the one hand, the real sizes of transcripts are unknown, because we did not analyze untranslated regions. On the other hand, the Northern analysis was performed with a probe that does not cover the newly discovered exons and may thus be incomplete.

The predicted protein products can be grouped into isoforms lacking completely the Dlg-A domains (e.g., LD33841), proteins similar to SAP97/hDLG that comprise both S97N and Dlg-A domains, and the well characterized Dlg-A lacking S97N. The first species was recently termed CPD (component protein of DLG) by Lee et al. (2002). These authors also reported on the expression of transcripts, which display similarity to Dlg-S97, although several differences in the use of exons in the DlgA-coding region are to be noted. The usage of alternative exons within this region provides a source for additional diversity within the Dlg protein family. Alternative splicing has also been reported for the mammalian Dlg homologs, i.e., rat SAP97 and hDLG. These proteins include the S97N domain fused to Dlg-A-like domains (Lue et al., 1994; Müller et al., 1995). Small insertions (I1-I5) have been characterized that do not alter the general protein structure $(\mathrm{Wu}$ et al., 2000; McLaughlin et al., 2002). The insertions are localized in the same regions in which alternatively spliced exons are detected in Dlg, i.e., in front of PDZ1 and in the HOOK region between SH3 and GUK domains. In contrast, in C. elegans, only a single form of DLG, which includes the Dlg-A domains and an N-terminal domain with $30 \%$ identity to $\mathrm{S} 97 \mathrm{~N}$, has been identified (Bossinger et al., 2001; Firestein and Rongo, 2001).

\section{S97N-containing proteins are predominantly expressed in nervous tissue and muscle}

Previous studies on Dlg expression and function used antibodies against the SH3-GUK (Woods and Bryant, 1991) or the PDZ1PDZ2 regions (Koh et al., 1999). On the basis of these analyses, Dlg-A was considered as the only $\mathrm{dlg}$ product that is expressed in epithelial cells, neurons, and muscle. On the basis of our studies, SH3-GUK antibodies should recognize at least three different isoforms, i.e., Dlg-A, the protein encoded by RE30579 and DlgS97, and anti-PDZ antibodies should detect Dlg-A, Dlg-S97, and proteins represented by RE30579 and GH01107. Antibodies against the $\mathrm{S} 97 \mathrm{~N}$ domain also may recognize a set of at least four predicted $d l g$ products, i.e., Dlg-S97 and proteins encoded by RE30579, GH01107, and LD33841. However, in combination with the previously described antibodies, they allowed a much better assignment of Dlg isoforms to cell types and developmental stages. Focusing on embryonic and larval development, we showed that $\mathrm{S} 97 \mathrm{~N}$-containing forms are already expressed in the cellular blastoderm stage, probably synthesized from maternal transcripts. From embryonic stage 11 onward, S97N immunoreactivity is restricted to neural tissue and at later stages to muscle cells. In contrast, anti-Dlg $\operatorname{PDZ1-2}_{2}$ antibodies stain both the developing neural system and epithelia. The RNA interference experiments showed that $\mathrm{S} 97 \mathrm{~N}$ immunoreactivity in the ventral cord disappeared when S97N-encoding transcripts were selectively targeted, whereas Dlg $\operatorname{lPZZ1-2}_{2}$-positive staining in epithelial cells persisted. Together, these results are consistent with the assumption that $\mathrm{S} 97 \mathrm{~N}$-containing proteins are not expressed in epithelia but are specifically present in neurons and muscle, in which they may constitute the prevalent Dlg isoforms.

\section{Potential roles of the S97N domain}

Detailed studies have addressed the functional roles of the different domains of Dlg-A and SAP97/hDLG (Hough et al., 1997; Wu et al., 1998, 2000; Thomas et al., 2000). The S97N domain can, for example, modulate the association of SAP97 with GKAP (guanylate kinase-associated protein)/SAPAPs (synapse-associated protein-associated proteins), one family of its cellular binding partners (Wu et al., 2000). In epithelia, $\mathrm{S} 97 \mathrm{~N}$ is required for recruitment of SAP97 to the cytoskeleton associated with the lateral membrane (Wu et al., 1998). This interaction is mediated by the L27 domain of Lin-2/CASK (calmodulin-dependent serine protein kinase) (Lee et al., 2002). An L27 motif is also present within the S97N domains of Dlg, SAP97/hDLG, and of a novel isoform of SAP90/PSD-95 (Marfatia et al., 2000; Chetkovich et al., 2002). In the latter case, it has been shown that L27 motifs may act as determinants for synaptic targeting and synaptic scaffold formation (Chetkovich et al., 2002). L27 domains of different proteins can interact with each other to form homo-oligomeric (i.e., hDLG) (Marfatia et al., 2000) or hetero-oligomeric protein complexes (Doerks et al., 2000). Consistently, S97N domains may be involved in scaffolding functions of Drosophila Dlg isoforms.

\section{Functional role of $d l g$ products}

The products of the $d l g$ gene participate in many processes. In epithelia, there is ample evidence for a role in the establishment of cellular polarity and intercellular adhesion (Tepass et al., 2001). Studies on Drosophila neurons have been focused on their implication in the organization and function of the larval NMJ. Dlg is present presynaptically and postsynaptically in type I synaptic boutons and is involved in the organization of the subsynaptic reticulum and in the regulation of neurotransmitter release (Lahey et al., 1994; Budnik et al., 1996). PDZ domains 1 and 2 bind Shaker potassium channels and mediate their synaptic localization (Tejedor et al., 1997). A similar function for Dlg can be assumed for the adult CNS (Ruiz-Cañada et al., 2002). Additionally, Dlg is fundamental for the synaptic localization of Fas II (Thomas et al., 1997a; Zito et al., 1997) and is involved in the regulation of synaptic plasticity (Koh et al., 1999). Our results suggest that S97N-containing isoforms, in particular Dlg-S97, may significantly contribute to the synaptic functions of Dlg. In this context it should be noted that this new information does not invalidate conclusions from previous works, because binding to Fas II and Shaker as well as synaptic targeting of Dlg proteins require domains of Dlg-A that are also present in Dlg-S97 (Thomas et al., 2000)

Immunostainings suggested that the $d l g$ mutants investigated here display reduced Dlg immunoreactivity in NMJ syn- 
aptic boutons compared with wild type, whereas labeling in the brain is not significantly diminished. This could be attributable to the expression of either naturally occurring or truncated Dlg isoforms containing S97N, PDZ1, and PDZ2 domains. The molecular data for $d l^{m 52}$ and $d l g^{X I-2}$ mutants predict truncated proteins, in front of the PDZ3 domain and the GUK domain, respectively. These studies confirm that none of the available mutants is a null mutant for the $d l g$ locus. This fact should be kept in mind when assessing these mutants for $d l g$ functions.

The expression of $597 \mathrm{~N}$-containing $d l g$ products and their extrasynaptic localization suggest that these isoforms serve additional roles during development. Indeed, severe defects in early neurogenesis were noticed in embryos lacking maternal and zygotic components of Dlg (Perrimon, 1988). Thus, $d l g$ products participate in the basal targeting of proteins involved in the establishment of asymmetric cell division in neuroblasts and sensory organ precursors (Ohshiro et al., 2000; Peng et al., 2000; Bellaiche et al., 2001). This is consistent with both the Perrimon (1988) data and our RNA interference experiments. The observed phenotype of embryos injected with S97N-dsRNA is not as severe as the phenotype obtained with the complete depletion of maternal and zygotic Dlg, confirming that $\mathrm{S} 97 \mathrm{~N}$-containing isoforms are involved in only part of the functions ascribed to $\mathrm{dlg}$. Nevertheless, severe defects in neurogenesis and neuronal differentiation in the PNS and CNS are associated with the inhibition of the expression of $597 \mathrm{~N}$-containing proteins in the embryo. The extent and variability of these defects may result from the perturbation of the asymmetric localization of cell fate determinants and/or from the disruption of subsequent differentiation, in which the role of $d l g$ products has not been understood yet.

\section{References}

Bellaiche Y, Radovic A, Woods DF, Hough CD, Parmentier ML, O'Kane CJ, Bryant PJ, Schweisguth F (2001) The partner of Inscuteable/Discs-large complex is required to establish planar polarity during asymmetric cell division in Drosophila. Cell 106:355-366.

Bossinger O, Klebes A, Segbert C, Theres C, Knust E (2001) Zonula adherens formation in Caenorhabditis elegans requires dlg-1, the homologue of the Drosophila gene discs large. Dev Biol 230:29-42.

Budnik V, Koh Y-H, Guan B, Haugh C, Woods D, Gorczyca M (1996) Regulation of synapse structure and function by the Drosophila tumor suppressor gene, dlg. Neuron 17:627-640.

Chetkovich DM, Bunn RC, Kuo S-H, Kawasaki Y, Kohwi M, Bredt DS (2002) Postsynaptic targeting of alternative postsynaptic density- 95 isoforms by distinct mechanisms. J Neurosci 22:6415-6425.

Doerks T, Bork P, Kamberov E, Makarova O, Muecke S, Margolis B (2000) L27, a novel heterodimerization domain in receptor targeting proteins Lin-2 and Lin-7. Trends Biochem Sci 25:317-318.

Ellis MC, O’Neill EM, Rubin GM (1993) Expression of Drosophila glass protein and evidence for negative regulation of its activity in non-neuronal cells by another DNA binding protein. Development 119:855-865.

Firestein BL, Rongo C (2001) DLG-1 Is a MAGUK similar to SAP97 and is required for adherens junction formation. Mol Biol Cell 12:3465-3475.

Garner CC, Nash J, Huganir RL (2000) PDZ domains in synapse assembly and signalling. Trends Cell Biol 10:274-280.

Grenningloh G, Rehm J, Goodman CS (1991) Genetic analysis of growth cone guidance in Drosophila: Fasciclin II functions as a neuronal recognition molecule. Cell 67:45-57.

Hough CD, Woods DF, Park S, Bryant PJ (1997) Organizing a functional junctional complex requires specific domains of the Drosophila MAGUK Discs large. Genes Dev 11:3242-3253.

Hummel T, Krukkert K, Roos J, Davis G, Klambt C (2000) Drosophila Futsch/ $22 \mathrm{C} 10$ is a MAP1B-like protein required for dendritic and axonal development. Neuron 26:357-370.
Kennerdell JR, Carthew RW (1998) Use of dsRNA-mediated genetic interference to demonstrate that frizzled and frizzled 2 act in the wingless pathway. Cell 95:1017-1026.

Koh YH, Popova E, Thomas U, Griffith LC, Budnik V (1999) Regulation of DLG localization at synapses by CaMKII-dependent phosphorylation. Cell 98:353-363.

Lahey T, Gorczyca M, Jia X, Budnik V (1994) The Drosophila tumor suppressor gene $d l g$ is required for normal synaptic bouton structure. Neuron 13:823-835.

Lee S, Fan S, Makarova O, Straight S, Margolis B (2002) A novel and conserved protein-protein interaction domain of mammalian Lin-2/CASK binds and recruits SAP97 to the lateral surface of epithelia. Mol Cell Biol 22:1778-1791.

Lue RA, Marfatia SM, Branton D, Chishti AH (1994) Cloning and characterization of hdlg: the human homologue of the Drosophila discs large tumor suppressor binds to protein 4.1. Proc Natl Acad Sci USA 91:9818-9822.

Marfatia SM, Byron O, Campbell G, Liu SC, Chishti AH (2000) Human homologue of the Drosophila discs large tumor suppressor protein forms an oligomer in solution. Identification of the self-association site. J Biol Chem 275:13759-13770.

McLaughlin M, Hale R, Ellston D, Gaudet S, Lue RA, Viel A (2002) The distribution and function of alternatively spliced insertions in $\mathrm{hDlg}$. J Biol Chem 277:6406-6412.

Mathew D, Gramates LS, Packard M, Thomas U, Bilder D, Perrimon N, Gorczycal M, Budnik V (2002) Recruitment of Scribble to the synaptic scaffolding complex requires GUK-holder, a novel DLG binding protein. Curr Biol 12:531-539.

Müller BM, Kistner U, Veh RW, Cases-Langhoff C, Becker B, Gundelfinger ED, Garner CC (1995) Molecular characterization and spatial distribution of SAP97, a novel presynaptic protein homologous to SAP90 and the Drosophila discs-large tumor suppressor protein. J Neurosci 15:2354-2366.

Myers EW, Sutton GG, Delcher AL, Dew IM, Fasulo DP, Flanigan MJ, Kravitz SA, Mobarry CM, Reinert KH, Remington KA, Anson EL, Bolanos RA, Chou HH, Jordan CM, Halpern AL, Lonardi S, Beasley EM, Brandon RC, Chen L, Dunn PJ, et al. (2000) A whole-genome assembly of Drosophila. Science 287:2196-2204.

Ohshiro T, Yagami T, Zhang C, Matsuzaki F (2000) Role of cortical tumoursuppressor proteins in asymmetric division of Drosophila neuroblast. Nature 408:593-596.

Peng C, Manning L, Albertson R, Doe CQ (2000) The tumour suppressor genes $l g l$ and $d l g$ regulate basal protein targeting in Drosophila neuroblasts. Nature 408:596-600.

Perrimon N (1988) The maternal effect of lethal(1)discs-large-1: a recessive oncogen of Drosophila melanogaster. Dev Biol 127:392-407.

Rechsteiner M, Rogers SW (1996) PEST sequences and regulation by proteolysis. Trends Biochem Sci 21:267-271.

Robinow S, White K (1991) Characterization and spatial distribution of the ELAV protein during Drosophila melanogaster development. J Neurobiol 22:443-461.

Ruiz-Cañada C, Koh Y-H, Budnik V, Tejedor FJ (2002) DLG differentially localizes Shaker $\mathrm{K}^{+}$-channels in the central nervous system and retina of Drosophila. J Neurochem 82:1490-1501.

Seeger M, Tear G, Ferres-Marco D, Goodman CS (1993) Mutations affecting growth cone guidance in Drosophila: genes necessary for guidance toward or away from the midline. Neuron 10:409-415.

Tejedor FJ, Bokhari A, Rogero O, Gorczyca M, Zhang J, Kim E, Sheng M, Budnik V (1997) Essential role for dlg in synaptic clustering of shaker K channels in vivo. J Neurosci 17:152-159.

Tepass U, Tanentzapf G, Ward R, Fehon R (2001) Epithelial cell polarity and cell junctions in Drosophila. Annu Rev Genet 35:747-784.

Thomas U, Kim E, Kuhlendahl S, Koh YH, Gundelfinger ED, Sheng M, Garner CC, Budnik V (1997a) Synaptic clustering of the cell adhesion molecule fasciclin II by discs-large and its role in the regulation of presynaptic structure. Neuron 19:787-799.

Thomas U, Phannavong B, Müller B, Garner CC, Gundelfinger ED (1997b) Functional expression of rat synapse-associated proteins SAP97 and SAP102 in Drosophila dlg-1 mutants: effects on tumor suppression and synaptic bouton structure. Mech Dev 62:161-174. 
Thomas U, Ebitsch S, Gorczyca M, Koh YH, Hough CD, Woods DF, Gundelfinger ED, Budnik V (2000) Synaptic targeting and localization of discs-large is a stepwise process controlled by different domains of the protein. Curr Biol 10:1108-1117.

Woods DF, Bryant PJ (1989) Molecular cloning of the lethal(1)discs large-1 oncogene of Drosophila. Dev Biol 134:222-235.

Woods DF, Bryant PJ (1991) The discs-large tumor suppressor gene of Drosophila encodes a guanylate kinase homolog localized at septate junctions. Cell 66:451-464.

Woods DF, Hough C, Peel D, Callaini G, Bryant PJ (1996) Dlg protein is required for junction structure, cell polarity, and proliferation control in Drosophila epithelia. J Cell Biol 134:1469-1482.
Wu H, Reuver SM, Kuhlendahl S, Chung WJ, Garner CC (1998) Subcellular targeting and cytoskeletal attachment of SAP97 to the epithelial lateral membrane. J Cell Sci 111:2365-2376.

Wu H, Reissner C, Kuhlendahl S, Coblentz B, Reuver S, Kindler S, Gundelfinger ED, Garner CC (2000) Intramolecular interactions regulate SAP97 binding to GKAP. EMBO J 19:5740-5741.

Zipursky SL, Venkatesh TR, Teplow DB, Benzer S (1984) Neuronal development in the Drosophila retina: monoclonal antibodies as molecular probes. Cell 36:15-26.

Zito K, Fetter RD, Goodman CS, Isacoff EY (1997) Synaptic clustering of Fascilin II and Shaker: essential targeting sequences and role of Dlg. Neuron 19:1007-1016. 